\title{
Study Protocol for a Multicenter, Open-Label, Single-Arm Study of Tranilast for Cardiomyopathy of Muscular Dystrophy
}

\author{
TSUYOSHI MATSUMURA ${ }^{1 *}$, HIROYA HASHIMOTO ${ }^{2 *}$, MASAHIRO SEKIMIZU'², ${ }^{2,}$ AKIKO M. SAITO ${ }^{2}$, \\ YUKO IWATA ${ }^{4}$, MASANORI ASAKURA ${ }^{5}$, KOICHI KIMURA ${ }^{6}$, TAKUHISA TAMURA ${ }^{7}$, \\ MICHINORI FUNATO ${ }^{8}$, KAZUHIKO SEGAWA ${ }^{9}$, KATSUHISA OGATA ${ }^{7}$ \\ AND TAKASHI NAKAJIMA ${ }^{10}$
}

\begin{abstract}
'Department of Neurology, National Hospital Organization Osaka Toneyama Medical Center, Toyonaka 560-8552, ${ }^{2}$ Clinical Research Center,

${ }^{3}$ Department of Pediatrics, National Hospital Organization Nagoya Medical Center, Nagoya 460-0001, ${ }^{4}$ Department of Clinical Research and Development, National Cerebral and Cardiovascular Center, Suita 565-8565, ${ }^{5}$ Department of Internal Medicine, Cardiovascular Division, Hyogo College of Medicine, Nishinomiya 663-8501, ${ }^{6}$ Department of General Medicine, The Institute of Medical Science, The University of Tokyo, Minato-ku 108-8639, ${ }^{7}$ Department of Neurology, National Hospital Organization Higashisaitama National Hospital, Hasuda 349-0196, ${ }^{8}$ Department of Pediatrics, Nagara Medical Center, Gifu 502-8558, ${ }^{9}$ Department of Cardiology, National Center Hospital, National Center of Neurology and Psychiatry, Kodaira 187-8551, ${ }^{10}$ Department of Clinical research, Department of Neurology, Niigata National Hospital, Kashiwazaki 945-8585, Japan *These authors contributed equally to the protocol development of this study.
\end{abstract}

Received 3 March 2019, accepted 15 April 2019

J-STAGE advance publication 15 June 2021

Edited by TAKAYUKI TANIWAKI

\begin{abstract}
Summary: Duchenne (DMD) and other forms of muscular dystrophy (MD) are collectively rare and affect approximately 20 per 100,000 people. The on-going development of exon skipping and other novel therapies for DMD is expected to lead to improvements in motor function prognosis. However, improvements in motor dysfunction with these novel therapies are associated with the risk of increase in cardiac burden. Development of therapies to improve cardiac function, therefore, is an urgent issue. This single-arm, open-label, multicenter study will include 20 patients with MD aged 13 years or older. Tranilast, a transient receptor potential cation channel subfamily V member 2 (TRPV2) inhibitor, will be administered orally for a period of 28 weeks at a dose of $300 \mathrm{mg} / \mathrm{day}$ divided into three daily doses. If consent to continue administration is obtained at 28 weeks, the drug will be administered for an additional 116 weeks. The primary outcome will be the change in brain natriuretic peptide (BNP) at 6 months after the start of administration compared to baseline. Tranilast is an anti-allergy agent that was developed in Japan. It has been used in a large number of clinical cases, including pediatric cases, and has been shown to be safe. We expect this study to provide basic data for developing new treatment method in cardiomyopathy/skeletal myopathy using TRPV2 inhibitors. Moreover, such therapies may also be effective in treating general heart failure without MD. Therefore, if the effectiveness of TRPV2 inhibitors could be confirmed in this study, great social and economic benefits could be achieved.
\end{abstract}

Key words muscular dystrophy, cardiomyopathy, tranilast, TRPV2 inhibitor

Corresponding Author: Hiroya Hashimoto, Ph.D., Clinical Research Center, National Hospital Organization Nagoya Medical Center, 4-1-1, Sannomaru, Naka-ku, Nagoya, Aichi 460-0001, Japan. Tel: +81-52-951-1111, Fax: +81-52-212-7360, E-mail: hiroya.hashimoto@nnh.go.jp 


\section{INTRODUCTION}

Duchenne muscular dystrophy (DMD) and other forms of muscular dystrophy (MD) are rare diseases that affect approximately 20 per 100,000 people. MD includes a large number of subtypes, including DMD, Becker muscular dystrophy, limb-girdle muscular dystrophy, and congenital muscular dystrophy. In addition to motor dysfunction, most of these dystrophies cause respiratory muscle dysfunction and myocardial dysfunction associated with respiratory and cardiac failures.

Artificial respiration has improved the vital prognosis of MD in many patients [1,2]. As a result, cardiac failure is still the most serious medical issue in MD. Angiotensin-converting-enzyme (ACE) inhibitor [3] and beta-blockers [3,4] have been employed in myocardial protection therapy, and such treatments have been successful in decreasing the number of patients with dilated cardiomyopathy [1-6]. However, there are limitations to the efficacy of myocardial dysfunction therapies. Long-term circulatory insufficiency causes problems such as increased renal failure and mortality [7], and therefore myocardial dysfunction has a major negative impact on vital prognosis and quality of life (QOL).

Currently, novel treatments such as exon skipping are being developed for DMD. While these therapies are expected to improve the motor function prognosis, exon skipping therapy is not likely to effect myocardiopathy. There is concern that improvements in motor dysfunction with novel therapies might increase the risk of cardiac burden. Under these circumstances, it is an urgent task to develop therapies aimed at supporting cardiac function.

Continuously high levels of intracellular calcium play a major role in MD myocardial and skeletal muscle degeneration. It was revealed that high levels of intracellular calcium were related to the stretch-activated channel known as the transient receptor potential cation channel subfamily V member 2 (TRPV2) [8]. It may be possible to suppress myocardial degeneration by inhibiting TRPV2, which involves a different mechanism from conventional treatments [8-10]. TRPV2 antagonist can be used concurrently with conventional myocardial protection treatments. It is expected that TRPV2 inhibitor will be effective in treating MD and other neuromuscular diseases, as well as cardiomyopathy and heart failure.

We will conduct an open-label, single-arm study of the TRPV2 inhibitor tranilast as an additional therapy in MD patients with heart failure and brain natriu- retic peptide (BNP) levels $\geq 100 \mathrm{pg} / \mathrm{mL}$ during standard cardiac protection therapy. BNP is a standard cardiac function indicator and is known to be highly correlated with heart failure severity and life prognosis. In MD, BNP $100 \mathrm{pg} / \mathrm{mL}$ or more is considered to be a predictive risk factor of death [11]. Our aim is to clarify whether tranilast can improve cardiac function by decreasing BNP level.

\section{METHODS}

\section{Study Design}

This is a single-arm, open-label, multicenter study registered in the UMIN Clinical Trials Registry (UMIN-CTR, registration number: UMIN000031965, URL: http://www.umin.ac.jp/ctr/) and the Japan Registry of Clinical Trials (jRCT, registration number: jRCTs031180038, URL: https://jrct.niph.go.jp/). An overview of this study is shown in Figure 1.

\section{Eligibility criteria}

Patients who satisfied the eligibility criteria shown in Table 1 will be enrolled in this study.

\section{Intervention}

Tranilast will be administered orally $100 \mathrm{mg}$ thrice daily for 28 weeks. Patients will continue to receive tranilast for 116 weeks additionally if they provide consent at 28 weeks. The observation and examination schedule is shown in Table 2.

Tranilast will be administrated orally but the simple suspension method is approved for patients using a feeding tube.

\section{Endpoints \\ Primary endpoint}

Changes in BNP levels for the six-month period (mean data at 20,24, and 28 weeks) from the baseline.

\section{Secondary endpoints}

1) Cardiac events: change in oral medications for cardiac failure or intravenous medication due to worsening in cardiac function, or hospitalization or prolongation due to heart failure.

2) Total mortality.

3) Left ventricle fractional shortening (FS).

4) Human atrial natriuretic peptide (hANP) and cardiac troponin $\mathrm{T}$ (cTnT) level.

5) TRPV2 expression on peripheral blood mononuclear cell (PBMC) surfaces.

6) Pinch strength and creatine kinase (CK) level.

7) Muscular dystrophy quality of life-60 (MDQoL-60) 
and the short form 12 health survey (SF-12).

8) Adverse events.

\section{Data collection and management}

We will use EDC to collect patient data. The principal investigator or individuals designated by the principal investigator will be logged into the EDC system using individual electronic signatures (ID and password) that are carefully stored. The collected case data will be entered into the EDC system and transmitted as soon as possible. The transmitted electronic data will serve as the case reports.

\section{Monitoring}

Central monitoring will be conducted using the data collected via the EDC system. As a general rule, monitoring via in-person visits to the facility will not be conducted. The data manager for this study will use central monitoring via the EDC system to monitor any suspicious data entered in the system whenever it is necessary. A scheduled monitoring report on the progress of the study will be issued once per year.

\section{Sample size}

The mean change in BNP after logarithm conversion $(\triangle \log [\mathrm{BNP}])$ in two cases included in a pilot study of tranilast administration was -0.18 , and the mean $\Delta \log (\mathrm{BNP})$ in 9 cases of MD (the control group in a multi-center trial of Carvedilol [5]) was 0.18 (standard deviation of 0.39). Based on our assumption that the $\Delta \log (\mathrm{BNP})$ would conform to the normal distribution with a mean of -0.18 and standard deviation of 0.39 , we calculated that the required number of patients would be 15 when performing the paired t-test on the null hypothesis (where the mean population $\Delta \log [\mathrm{BNP}]$ is 0.18 ) with the significance level of $5 \%$ and power of $90 \%$. In consideration of the possibility of drop-outs, we then set the number of patients at 20 .

"The $\Delta \log (\mathrm{BNP})$ is equal to -0.18 " means that the BNP geometric mean ratio is 0.66 ; i.e. there is a $34 \%$ decrease as compared to the pre-administration BNP level.

\section{Statistical analysis}

Efficacy analyses will be performed on the full analysis set (FAS) and additional analyses will be performed on the per protocol set (PPS) to evaluate the sensitivity. The FAS consists of all enrolled patients without any critical protocol violation including failure in consent acquisition. The PPS includes patients in the FAS without any major protocol deviations. The safety analysis set is defined as all enrolled patients receiving at least one dose of tranilast.

All statistical tests will be conducted with a twosided significance level of 5\%. Analysis of the primary end point consists of calculating the geometric mean, geometric standard deviation, median, and the interquartile range, and the paired t-test will be performed on the amount of change in the logarithmic conversions. The null hypothesis stating that the mean population $\Delta \log (\mathrm{BNP})$ is 0.18 will be tested.

In the case of conducting the test on the secondary endpoints, we will target only the data at 24 weeks. For time to cardiac events and total mortality, survival
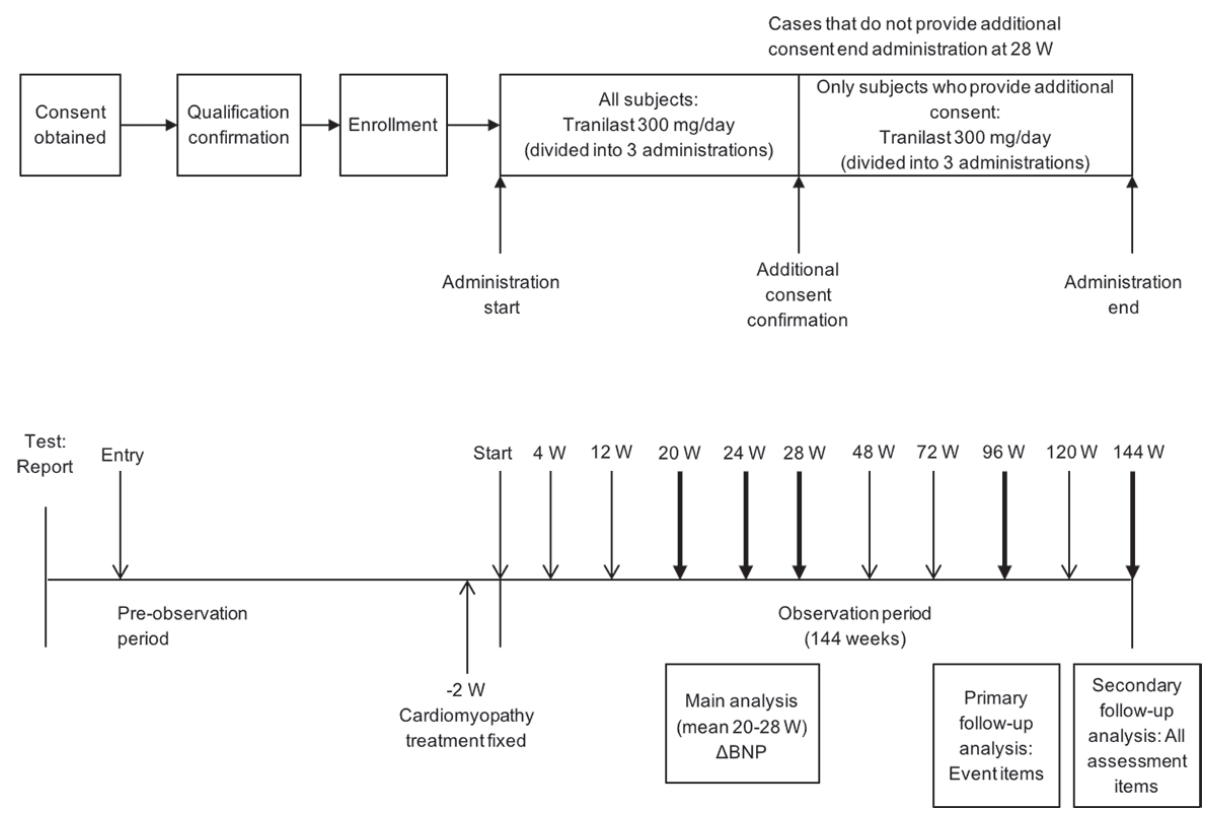

Fig. 1. Study overview. 
curves will be estimated using the Kaplan-Meier method. Summary statistics for FS, TRPV2 expression, pinch strength, MDQoL-60, and SF-12 will be calculated, and the paired t-test will be used on the amount of change. Furthermore, hANP, cTnT, and CK will be examined by calculating the geometric mean and geometric standard deviation, and the paired t-test will be performed on the amount of change in the logarithmic conversions. The frequency of adverse events will be calculated.

\section{Analysis period}

The main analyses of the primary and secondary end points and safety will be conducted when BNP data at 20,24 and 28 weeks and other examination and observation data at 24 weeks for all patients are obtained.

The founder will determine based on the results of these main analyses whether the study will be continued or stopped. If the study is determined to continue, the primary follow-up analysis will be conducted for the cardiac events, total mortality, and adverse events at 96 weeks. If the study continues beyond 96 weeks, all data will be analyzed at the 144-week time point.

Statistical tests for each end point will be performed once in order to avoid multiple testing problems.

\section{Study organization}

Fifteen institutions in Japan will participate in this study. The Data and Safety Monitoring Committee consists of two cardiovascular specialists and a neurologist.

\section{DISCUSSION}

Tranilast is an anti-allergic agent which was developed in Japan and has been on the market for over 30 years. It has been prescribed to many adults and children. It is expected to be safe to administer a $300 \mathrm{mg}$ daily dose of tranilast, as that dosage is already approved by the National Health Insurance system. It is confirmed that tranilast inhibits TRPV2 [14]. Therefore, this drug is an appropriate compound to assess

TABLE 1.

Eligibility Criteria

\section{Inclusion criteria}

1. MD patients aged $\geq 13$ years.

2. BNP level of $\geq 100 \mathrm{pg} / \mathrm{mL}$ at pre-observation.

3. Patients who are using the standard myocardial protective agents (ACEI/ARB and/or beta-blockers) and who satisfy both of the following:

Taking maintenance-level doses at the time of consent to participate in this study.

Taking a fixed dose using a fixed administration method from two weeks prior to the start of administration of the test drug in this study until the start of administration of the test drug.

4. Patients who are able to ingest capsules or granules/granules in dry syrup, or patients able to undergo administration via feeding tube.

5. Patients or their legal guardians from whom written consent was granted of their own free will.

\section{Exclusion criteria}

1. Acute cardiac failure (undergoing intravenous cardiotonic, diuretic, or anti-arrhythmia drug treatment).

2. Undergoing non-fixed doses or administration methods of digitalis, diuretics, aldosterone antagonist, cardiotonics, antiarrhythmia drugs during the period from two weeks prior to the start of administration until the start of administration.

3. Presence of lethal arrhythmia (e.g. $\geq 4$ continuous premature ventricular contractions). Except for patients with implantable cardioverter defibrillators.

4. Severe renal dysfunction (eGFR using cystatin c of under $30 \mathrm{~mL} / \mathrm{min} / 1.73 \mathrm{~m}^{2}$ [12]). In the case of patients under the age of 18 years, cystatin $\mathrm{c}$ of $\geq 2.4 \mathrm{mg} / \mathrm{L}$.

5. Severe liver dysfunction (T. Bil $\geq 10 \mathrm{mg} / \mathrm{dL}, \mathrm{AST} / \mathrm{ALT} \geq 500 \mathrm{IU} / \mathrm{L}, \mathrm{ALP} \geq 5 \times$ normal max. limit, PT $\leq 40 \%$, liable to hemorrhage, hepatic insufficiency with disturbance of consciousness [fulminant hepatitis], cirrhosis of the liver, liver tumor jaundice persisting for $\geq 6 \mathrm{M}$, Grade 3 according to the "Severity Criteria for Adverse Effects of Pharmaceuticals and other Agents" [13].

6. Marked leukopenia (under $3000 / \mu \mathrm{L}$ ), thrombocytopenia (under $80000 / \mu \mathrm{L}$ )

7. History of hypersensitivity to tranilast.

8. Pregnant women or those who may be pregnant.

9. Any case in which the principal investigator or sub-investigator(s) has determine to be inappropriate for participation in this study.

MD, muscular dystrophy; BNP, brain natriuretic peptide. 
TABLE 2 .

Observation \& testing schedule.

\begin{tabular}{|c|c|c|c|c|c|c|c|c|c|c|}
\hline \multirow{2}{*}{\multicolumn{2}{|c|}{$\begin{array}{l}\text { Item } \\
\\
\text { Time period } \\
\text { (W; weeks) }\end{array}$}} & $\begin{array}{l}\text { Pre-obser- } \\
\text { vation }\end{array}$ & $\begin{array}{c}\text { Day of } \\
\text { administra- } \\
\text { tion start }\end{array}$ & \multicolumn{5}{|c|}{ Short-term administration period ${ }^{* 1}$} & \multicolumn{2}{|c|}{$\begin{array}{l}\text { Long-term } \\
\text { administration } \\
\text { period }^{* 2}\end{array}$} \\
\hline & & Pre-2-8 W & $0 \mathrm{~W}$ & $4 \mathrm{~W}$ & $12 \mathrm{~W}$ & $20 \mathrm{~W}$ & $\begin{array}{c}24 \mathrm{~W} \\
\text { or } \\
\text { discon- } \\
\text { tinuation }\end{array}$ & $28 \mathrm{~W}$ & $\begin{array}{c}36 \mathrm{~W} \\
60 \mathrm{~W} \\
84 \mathrm{~W} \\
108 \mathrm{~W} \\
132 \mathrm{~W}\end{array}$ & $\begin{array}{l}48 \mathrm{~W}, \\
72 \mathrm{~W}, \\
96 \mathrm{~W} \\
120 \mathrm{~W}, \\
144 \mathrm{~W}\end{array}$ \\
\hline \multicolumn{2}{|c|}{ Consent obtained } & $\bullet$ & & & & & & $\bullet^{\mathrm{h}}$ & & \\
\hline \multicolumn{2}{|c|}{$\begin{array}{l}\text { Patient background } \\
\text { characteristics }\end{array}$} & $\bullet$ & & & & & & & & \\
\hline \multicolumn{2}{|c|}{ Test drug administration } & & & \multicolumn{7}{|c|}{ <-----------------------------------------------------------> } \\
\hline \multicolumn{2}{|c|}{$\begin{array}{l}\text { Subjective symptoms/ } \\
\text { objective symptoms }\end{array}$} & $\bullet$ & $\bullet$ & $\bullet$ & $\bullet$ & $\bullet$ & $\bullet$ & $\bullet$ & $\bullet$ & $\bullet$ \\
\hline \multicolumn{2}{|c|}{ Observation of adverse event(s) } & & & \multicolumn{7}{|c|}{ 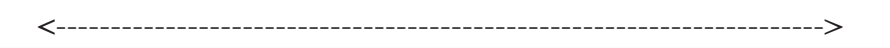 } \\
\hline \multicolumn{2}{|c|}{ Vital signs measurement } & $\bullet$ & $\bullet$ & $\bullet$ & $\bullet$ & $\bullet$ & $\bullet$ & $\bullet$ & $\bullet$ & $\bullet$ \\
\hline \multicolumn{2}{|c|}{ Motor function measurement } & & $\bullet$ & & $\bullet$ & & $\bullet$ & & & $\bullet$ \\
\hline \multirow{3}{*}{$\begin{array}{l}\text { Clin- } \\
\text { ical } \\
\text { Tests }\end{array}$} & Hematology examination $^{\mathrm{b}}$ & $\bullet$ & $\bullet$ & $\bullet$ & $\bullet$ & $\bullet$ & $\bullet$ & $\bullet$ & & $\bullet$ \\
\hline & $\begin{array}{l}\text { Blood } \\
\text { biochemical examination }^{c}\end{array}$ & ○ & $\bullet$ & $\bullet$ & $\bullet$ & ○ & $\bullet$ & ० & & $\bullet$ \\
\hline & Urine test $^{\mathrm{d}}$ & ० & $\bullet$ & $\bullet$ & $\bullet$ & 0 & $\bullet$ & ० & & $\bullet$ \\
\hline \multicolumn{2}{|c|}{$\begin{array}{l}\text { Sample collection for: } \\
\text { TRPV2 expression on peripheral } \\
\text { blood mononuclear cell surface } \\
\text { miRNA profile } \\
\text { Metabolome analysis }^{\mathrm{e}}\end{array}$} & & $\bullet$ & $\bullet$ & $\triangle$ & & $\triangle$ & & & $\triangle$ \\
\hline \multicolumn{2}{|c|}{ 12-lead ECG } & & $\bullet$ & $\triangle$ & $\bullet$ & & $\bullet$ & & & $\bullet$ \\
\hline \multicolumn{2}{|c|}{ Holter ECG ${ }^{f}$} & $\bullet$ & ○ & $\triangle$ & $\triangle$ & & $\bullet$ & & & $\bullet$ \\
\hline \multicolumn{2}{|c|}{ Echocardiography } & & $\bullet$ & & $\triangle$ & & $\bullet$ & & & $\bullet$ \\
\hline \multicolumn{2}{|c|}{ Respiratory function measurement } & & $\bullet$ & & $\triangle$ & & $\bullet$ & & & $\bullet$ \\
\hline \multicolumn{2}{|c|}{ Plain chest X-ray } & & $\bullet$ & $\triangle$ & $\bullet$ & & $\bullet$ & & & $\bullet$ \\
\hline \multicolumn{2}{|c|}{ MDQoL-60/SF-12 } & & $\bullet$ & & & & $\bullet$ & & & $\bullet$ \\
\hline
\end{tabular}

$* 1$ : Permissible range of \pm 7 days. $\bullet / \circ$ indicates required item, $\triangle$ indicates optional item

$* 2$ : Conducted after receiving additional consent to continue administration at 28 weeks. Permissible range of \pm 4 weeks.

a) Adverse event observation period extends until the final day of administration.

b) Hematology test items: RBC, Hb, Ht, WBC, Eo, Plt (Pre-observation period data: Data obtained within 3 months prior to the initial medical examination may be used).

c) Blood biochemical test items: hANP, BNP, cTnT, $\underline{\mathrm{CK}}, \underline{\mathrm{AST}}, \underline{\mathrm{ALT}}, \underline{\gamma-\mathrm{GTP}}, \mathrm{LDH}, \mathrm{T} . \mathrm{Bil}, \mathrm{BUN}, \mathrm{Cr}, \mathrm{UA}, \underline{\mathrm{Cy} s t a t i n} \mathrm{C}, \mathrm{Na}, \mathrm{K}$, $\mathrm{Cl}$, IgE, lactate, pyruvate, ketone body fraction, Val, Leu, Ile ( $\bullet$ indicates "all items," $\circ$ indicates that underlined items are required, pre-observation period data: data obtained within 3 months prior to the initial medical examination may be used) The hANP, BNP, cTnT, cystatin C, ketone body fraction, Val, Leu, and Ile measurements to be performed by the same subcontracted laboratory.

d) Urine test items: protein, glucose, occult blood, $8-\mathrm{OHdG}$, titin ( $\circ$ indicates that underlined items are required, $\bullet$ indicates 8-OhdG also measured, titin is optional).

e) Seven milliliters of whole blood collected, TRPV2 testing: separate storage of peripheral blood mononuclear cells, miRNA profile, metabolome analysis: plasma storage.

f) Holter ECG: in cases in which data was obtained within 3 months prior to pre-observation period, re-testing at pre-observation period is unnecessary. In cases in which data was obtained within 3 months prior to the 0 week time point and no change was made in concomitant drugs affecting cardiac function, re-testing at 0 week time point is unnecessary.

g) Additional consent obtained at 48, 96, and 144 weeks.

h) At the 28-week time point, intent to continue participation in the study is to be reconfirmed and noted in the patient's medical records. 
the efficacy and safety of treatments aimed at inhibiting TRPV2.

We expect this study to provide basic data for developing new treatment methods in cardiomyopathy/ skeletal myopathy using TRPV2 inhibitors. Such therapies may also be effective in general heart failure without MD, and could bring great social and economic benefits.

\section{DECLARATION}

COMPETING INTERESTS: The authors declared that they have no competing interests.

FUNDING: This study is supported by Grant-in-Aid for Clinical Research from the National Hospital Organization.

AUTHORS' CONTRIBUTIONS: TM conceived this study and prepared an implementation plan. $\mathrm{HH}$ refined the study design and statistical analysis. MS, YI, MA, KK, TT, MF, KS, $\mathrm{KO}$ and TN participated in its design and coordination and helped to draft the manuscript. AMS supported the creation of the protocol on data management and monitoring. All authors reviewed and approved the manuscript.

ETHICS AND DISSEMINATION: This study will be conducted in accordance with the Declaration of Helsinki, the Clinical Trials Act (2017, no. 16), the Enforcement Regulations of the Clinical Trials Act (2018, Ministry of Health, Labour and Welfare, no. 17), and the Act on the Protection of Personal Information. This study was approved in October 2018 by the National Hospital Organization Review Board for Clinical Trials.

The principal investigator or sub-investigators will obtain written consent from the patients themselves or from their legal representative prior to their participation in the study. In cases in which a study participant is a legal minor (or a patient without sufficient ability to make judgment on his/her own), an explanation of the study description form will be provided to the study participant and legal representative and, in cases in which the study participant's level of comprehension is deemed suitable, an explanation will also be provided of the consent form. Consent to participate in this study will be obtained from the legal representative and, to the extent possible, from the patient him- or herself.

The results of this study are scheduled to be published within 2 year after the end of the study, by conference presentation or paper publication.

DATA SHARING: There is no data available.

\section{REFERENCES}

1. Matsumura T, Saito T, Fujimura H, Shinno S, and Sakoda S. A longitudinal cause-of-death analysis of patients with Duchenne muscular dystrophy. Rinsho Shinkeigaku 2011; 51:743-750. (in Japanese)

2. Ishikawa Y, Miura T, Ishikawa Y, Aoyagi T, Ogata H et al. Duchenne muscular dystrophy: survival by cardio-respiratory interventions. Neuromuscul Disord 2011; 21:47-51.

3. Tamura T, Shibuya M, Iida M, Ishihara D, Fukunaga H, et al. Clinical Evaluation of Captopril in Chronic Cardiac Dysfunction in Patients with Duchenne Muscular Dystrophy. Clin Med 1996; 12:3635-3646.

4. Ishikawa Y, Bach JR, Minami R. Cardioprotection for Duchenne's muscular dystrophy. Am Heart J 1999; 137:895-902.

5. Matsumura T, Tamura T, Kuru S, Kikuchi Y, and Kawai M. Carvedilol can Prevent Cardiac Events in Duchenne Muscular Dystrophy. Intern Med 2010; 49:1357-1363.

6. Kajimoto $\mathrm{H}$, Ishigaki $\mathrm{K}$, Okumura $\mathrm{K}$, Tomimatsu $\mathrm{H}$, Nakazawa $M$ et al. Beta-Blocker Therapy for Cardiac Dysfunction in Patients with Muscular Dystrophy. Circ J 2006; 70:991-994.

7. Matsumura T, Saito T, Fujimura H, and Sakoda S. Renal dysfunction is a frequent complication in patients with advanced stage of Duchenne muscular dystrophy. Clin Neurol 2012; 52:211-217.

8. Iwata Y, Katanosaka Y, Arai Y, Komamura K, Miyatake K et al. A novel mechanism of myocyte degeneration involving the $\mathrm{Ca} 2+-$ permeable growth factor-regulated channel. J Cell Biol 2003; 161:957-967.

9. Iwata Y, Katanosaka Y, Arai Y, Shigekawa M, and Wakabayashi S. Dominant-negative inhibition of $\mathrm{Ca} 2+$ influx via TRPV2 ameliorates muscular dystrophy in animal models. Hum Mol Genet 2009; 18:824-834.

10. Zanou N, Iwata Y, Schakman O, Lebacq J, Wakabayashi S et al. Essential role of TRPV2 ion channel in the sensitivity of dystrophic muscle to eccentric contractions. FEBS Lett 2009; 583:3600-3604.

11. Demachi J, Kagaya Y, Watanabe J, Sakuma M, Ikeda J et al. Characteristics of the increase in plasma brain natriuretic peptide level in left ventricular systolic dysfunction, associated with muscular dystrophy in comparison with idopathic dilated cardiomyopathy. Neuromuscul Disord 2004; 14:732-739.

12. Japanese Society of Nephrology, eds. CKD Guideline 2012. Tokyo Igakusha, 2012; 18-21. (in Japanese)

13. Drug Safety Notification. Notification No. 80 of the Safety Division Chief, Pharmaceutical Affairs Bureau, Ministry of Health and Welfare. "Classification criteria for the severity of adverse drug reactions." (in Ja-panese) http://www. mhlw.go.jp/shingi/2005/10/dl/s1006-4f2.pdf.

14. Iwata Y, Ohtake H, Suzuki O, Matsuda J, Komamura K, et al. Blockade of sarcolemmal TRPV2 accumulation inhibits progression of dilated cardiomyopathy. Cardiovasc Res 2013; 99:760-768. 